\title{
Estudo Comparativo entre Ferramentas baseadas em Conhecimento para a Gestão da Inovação
}

\author{
Carine G. Webber, Tiago Zattera da Silveira
}

\author{
Centro de Computação e TI - Universidade de Caxias do Sul (UCS) \\ Caixa Postal 15.064 - 91.501-970 - Caxias do Sul - RS - Brazil \\ \{cgwebber, tzsilvei\}@ucs.br
}

\begin{abstract}
Innovation is a strategic differential in a world of strong business competitiveness. An innovation is not just an idea but an elaborated process that results in the implementation of a new or significantly improved product or organizational process. Innovation comprises steps of generating ideas, resource allocation, implementation and acceptance by a consumer market. Software tools have an important role, supporting activities that enable or facilitate information sharing, acquisition and classification of ideas, research and data collection on a scientific basis and trends forecast. In this context, this work presents a comparison among main software tools applied to Innovation Management.
\end{abstract}

Resumo. A capacidade de inovar é um fator de diferenciação estratégica em contextos de acirrada competitividade empresarial. Uma inovação não é apenas uma ideia mas um processo elaborado que resulta na implementação de um novo ou significativamente melhorado produto ou processo organizacional. A inovação compreende etapas de geração de ideias, alocação de recursos, implementação e aceitação por um mercado consumidor. Ferramentas de Software tem um papel importante apoiando atividades que viabilizam ou facilitam a aplicação das metodologias, através do compartilhamento de informações, a aquisição e classificação de ideias, a pesquisa e coleta de dados em bases científicas e a previsão de tendências. Neste contexto, o presente estudo apresenta uma comparação entre as principais ferramentas de softwares inseridas em atividades da Gestão da Inovação, tais como a Gestão de Ideias e a Inovação Aberta.

\section{Introdução}

A Gestão da Inovação (GI) é um processo que compreende etapas de geração de ideias, alocação de recursos, implementação e aceitação por um mercado consumidor. Um dos fatores mais importantes em processos de Inovação é a capacidade de criar novos conhecimentos. Nonaka e Takeuchi (2008) defendem a empresa criadora de conhecimento, cujo negócio principal é a inovação constante, dando ênfase aos processos que agregam valor à informação e a disseminação do conhecimento. Segundo os autores, empresas bem sucedidas são as que criam constantemente novos conhecimentos e os incorporam rapidamente em novas tecnologias e produtos.

Um processo de inovação resulta na implementação de um novo ou significativamente melhorado produto ou processo organizacional. Segundo a OECD (2005) a adaptação ou utilização de softwares é necessária para o desenvolvimento e a implementação das inovações. A introdução da TI no suporte à inovação é destacada por diversos autores que afirmam que ela pode padronizar e organizar processos de inovação ou ainda caracterizam a adaptação ou utilização de softwares como necessária para o desenvolvimento e a implementação das inovações. As principais categorias de softwares são as seguintes: Gestão de Ideias, Inovação Aberta, Gestao do Conhecimento (GC), Prospecção e Busca Semântica. As ferramentas viabilizam ou facilitam processos de GI, através do compartilhamento de informações, a aquisição e classificação de ideias, a pesquisa e coleta de dados em bases científicas e a previsão de tendências. Nesta perspectiva, este artigo tem como objetivo apresentar um estudo sobre o uso de ferramentas de TI em processos de GI. Devido a 
extensa gama de soluções de TI, o estudo explorou somente softwares aplicados ao contexto da inovação que se encontram em uso por renomadas empresas inovadoras, uma vez que seria inviável aqui tratar de todos os softwares existentes no mercado.

\section{Metodologias de Gestão da Inovação}

De modo a incentivar e facilitar o processo de inovação organizacional, diversos autores propõem o uso de metodologias de inovação. As metodologias buscam reunir as melhores práticas de empresas inovadoras com características e etapas fundamentais. Para fins de estudo e avaliação foram selecionadas as metodologias TEMAGUIDE [COTEC 1998] e NUGIN [Lodi 2006]. A seleção seguiu critérios de aplicabilidade em organizações de diferentes portes ou segmentos, relevância mundial e aplicabilidade no cenário nacional.

A TEMAGUIDE é resultado de uma pesquisa realizada por um grupo de organizações europeias. No cenário internacional ela é vista como um das principais metodologias de GI [Cavalcanti 2009]. Ela propõe um conjunto de atitudes estratégicas que visam revisar e promover todos os assuntos favoráveis à inovação [Limas, Scandelari e Franciso, 2008]. A estrutura básica da metodologia contempla a Gestão Tecnológica e da Inovação através de uma perspectiva de negócios. Os elementos essenciais para o processo de inovação segundo o TEMAGUIDE são monitoramento (scan), focalização (focus), alocação de recursos (resource), implementação (implement) e aprendizado (learn). O modelo de inovação pode ser usado tanto num nível prático para gerir o processo de inovação quanto num nível estratégico para assegurar que a Gestão Tecnológica seja integrada aos negócios. O modelo considera ainda que para inovar com sucesso deve-se inovar com frequência.

A metodologia NUGIN promove uma visão sistêmica da organização, além da integração do nível estratégico, tático e operacional e das diferentes áreas da empresa, valorizando a comunicação e os relacionamentos. A NUGIN é uma metodologia de planejamento e GI que possibilita identificação de gargalos tecnológicos, criação de núcleos integrados de pesquisa e desenvolvimento, e implementação de ferramentas de gestão da inovação nas empresas [Lodi 2007, Cavalcanti 2009]. A implementação deste modelo envolve diversas etapas. Inicialmente é realizado o diagnóstico que visa identificar pontos fortes e fracos da empresa e a capacidade de inovação. A etapa seguinte é o planejamento estratégico da inovação. Analisadas as oportunidades oferecidas e definidas as estratégias necessárias, a etapa seguinte é o desenvolvimento de inovações. A última etapa proposta é de Inteligência Competitiva. Essa fase permeia todas as demais, com funções ao longo de todo o processo de inovação.

\section{Softwares aplicados à Gestão da Inovação}

Segundo a OECD (2005), a utilização de softwares é necessária para o desenvolvimento e a implementação das inovações. Nesse estudo são abordados apenas softwares que se enquadram em etapas propostas pelas metodologias TEMAGUIDE e NUGIN. Além disso, foi considerada a relevância das ferramentas no contexto da inovação e a utilização por empresas inovadoras. Como a quantidade de produtos está em constante desenvolvimento é importante frisar que seria inviável nesse estudo analisar-se todos eles. As categorias analisadas compreendem softwares de suporte à Gestão de Ideias, Inovação Aberta, Gestão do Conhecimento, Prospecção e Análise Semântica. Os seguintes softwares foram estudados: Idea Box, Innovation Suite, Accept 360 Ideation, Jenny, Idea Ken, Media Wiki, InWeb e Sphinx.

O software Idea Box representa uma caixa de sugestões onde os colaboradores podem publicar ideias inovadoras [Ideabox 2011]. Conjunto de softwares focados em GI, o Innovation Suite é composto pelos módulos Web Storm, Switchboard e Pipeline. Cada módulo representa parte de uma diversificada gama de serviços úteis no apoio à inovação, incluindo ferramentas que permitem a coleta e gerenciamento de ideias e o acompanhamento de projetos de inovação 
[BRIGHTIDEA 2011]. Com recursos para gerenciar ideias externas, realizar votações promovendo as ideias mais promissoras e divulgar as inovações produzidas eletronicamente, o Accept 360 Ideation permite coletar e avaliar ideias. O software permite ainda o envolvimento de clientes, parceiros e colaboradores [ACCEPT 2011].

Focado no conceito de Campanhas de Ideias, o software o Jenny Idea Management [JPB 2011] permite através de interface Web o registro de ideias de colaboradores, clientes e fornecedores coletadas através de brainstorms online. A ferramenta fornece ainda mecanismos para classificação do potencial de cada ideia e permite disponibilizar determinadas campanhas para um público alvo. Os softwares citados se enquadram na fase de focalização do TEMAGUIDE e no desenvolvimento das inovações modelado na metodologia NUGIN. Em ambas as etapas são previstas as atividades de avaliação e seleção das ideias que proporcionem maiores benefícios ou ofereçam maiores chances de sucesso para a definição de qual ideia será desenvolvida.

Uma ferramenta colaborativa para obtenção de ideias é o Idea Ken Innovation Management Tool. Voltado para empresas de médio e grande porte, funciona como uma plataforma de serviços na qual é possível definir um problema e estabelecer uma recompensa para ideias inovadoras que contribuam efetivamente com a solução [IDEAKEN 2011].

A Wiki pode ser utilizada nas etapa de monitoramento e aprendizagem da metodologia de inovação TEMAGUIDE ou ainda para apoiar atividades da NUGIN, auxiliando o comitê estratégico da inovação a obter informações sobre diferentes áreas da organização, ou no planejamento estratégico da inovação, onde se devem analisar os conhecimentos internos e externos da organização.

As ferramentas de TI para apoiar o processo de Prospecção possuem enfoque bastante diversificado e, em geral, não foram desenvolvidas especificamente para a inovação. Dentre os produtos, o INWEB é um observatório da Web que está sempre buscando novos algoritmos e tecnologias para aumentar a integração internet - sociedade [INWEB 2011]. Dessa forma, procura identificar, caracterizar e modelar interesses e padrões de comportamento das pessoas na Web. A partir disso é feito um processamento e tratamento das informações. Analisadas essas informações pode-se entender o que as pessoas buscam hoje para antecipar possibilidades de futuro. Outro software que pode ganhar destaque nesse tipo de aplicação é o Sphinx [SPHINX BRASIL 2010]. Através do Sphinx é possível coletar e classificar dados de textos e bases de dados específicas ou da Web. Além disso, é possível realizar pesquisas locais e via Internet. Para isso o software conta com técnicas de mineração dos dados e geração de relatórios através de estatísticas qualitativas e quantitativas. As ferramentas de prospecção podem ser aplicadas na etapa de monitoramento do TEMAGUIDE (auxiliando na procura de sinais que indiquem a necessidade de inovação ou setores que ofereçam oportunidades em potencial) e na fase de planejamento estratégico da inovação da metodologia NUGIN. Nessa fase são coletadas e analisadas informações do ambiente interno e externo que ajudam a traçar oportunidades de inovação.

Os softwares mencionados foram testados e avaliados conforme descrito na próxima seção.

\section{Método de Avaliação dos Softwares}

Para auxiliar a avaliação e atingir o objetivo proposto aplicou-se o Método Analítico Hierárquico (MAH) como instrumento para a tomada de decisões multicritério. Ele se destaca por aceitar variáveis quantitativas e qualitativas, de modo que até mesmo impressões subjetivas do tomador de decisão podem ser transformadas em notas lineares para a classificação das alternativas [Moraes e Santaliestra 2008].

A elaboração dos critérios utilizados neste trabalho se baseou em conceitos verificados em metodologias de GI (NUGIN e TEMAGUIDE), nas melhores práticas de empresas inovadoras e em agentes influenciadores da inovação. Apesar de o MAH possibilitar a definição de diferentes pesos para cada critério, a todos foi atribuído o mesmo peso. A tabela 1 apresenta os critérios definidos. 
Tabela 1: Critérios de Avaliação

\begin{tabular}{c|l}
\hline Legenda & Critério de Avaliação \\
\hline C1 & Suporte a aquisição de conhecimento \\
\hline C2 & Monitoramento e integração com ambiente interno / externo \\
\hline C3 & Priorização de propostas de projetos de inovação \\
\hline C4 & Avaliação da viabilidade de um projeto de inovação \\
\hline C5 & Capacidade de coleta e registro de ideias \\
\hline C6 & Compartilhamento e colaboração de ideias \\
\hline C7 & Reconhecimento pela autoria de ideias \\
\hline C8 & Suporte a alocação de recursos \\
\hline C9 & Mensurar gastos com inovação \\
\hline C10 & Registro de acertos e erros em projetos \\
\hline C11 & Medir difusão da inovação \\
\hline
\end{tabular}

Os seguintes softwares foram objeto da avaliação e comparação: Idea Box, Media Wiki, Accept 360 Ideation, Jenny, Innovation Suite, Idea Ken, InWeb e Sphinx. Foram cruzadas funcionalidades e características destes softwares com os critérios de avaliação propostos. A Tabela 2 apresenta esse cruzamento, de forma que cada linha representa um critério e cada coluna representa um software (sim-o software atende o critério, não-o software não atende o critério).

Tabela 2: Cruzamento entre softwares e critérios de avaliação

\begin{tabular}{|c|c|c|c|c|c|c|c|c|}
\hline $\begin{array}{l}\text { Critério de } \\
\text { Avaliação }\end{array}$ & Idea Box & Media Wiki & $\begin{array}{l}\text { Accept } 360 \\
\text { Ideation }\end{array}$ & Jenny & Innov. Suite & $\begin{array}{l}\text { Idea } \\
\text { Ken }\end{array}$ & InWeb & Sphinx \\
\hline C1 & NÃO & SIM & NÃO & NÃO & NÃO & NÃO & NÃO & NÃO \\
\hline C2 & NÃO & NÃO & SIM & SIM & SIM & SIM & SIM & SIM \\
\hline C3 & NÃO & NÃO & SIM & SIM & SIM & SIM & NÃO & NÃO \\
\hline C4 & NÃO & NÃO & SIM & SIM & SIM & SIM & NÃO & NÃO \\
\hline C5 & SIM & SIM & SIM & SIM & SIM & SIM & NÃO & NÃO \\
\hline C6 & SIM & SIM & SIM & SIM & SIM & SIM & NÃO & NÃO \\
\hline C7 & SIM & NÃO & NÃO & NÃO & SIM & SIM & NÃO & NÃO \\
\hline $\mathrm{C} 8$ & NÃO & NÃO & NÃO & NÃO & SIM & NÃO & NÃO & NÃO \\
\hline C9 & NÃO & NÃO & NÃO & SIM & NÃO & NÃO & NÃO & NÃO \\
\hline C10 & NÃO & NÃO & NÃO & NÃO & NÃO & NÃO & NÃO & NÃO \\
\hline C11 & NÃO & NÃO & NÃO & NÃO & NÃO & NÃO & NÃO & NÃO \\
\hline $\begin{array}{l}\text { Critérios } \\
\text { Atendidos }\end{array}$ & 3 & 3 & 5 & 6 & 7 & 6 & 1 & 1 \\
\hline
\end{tabular}

A próxima etapa no processo avaliativo realizou o cruzamento de cada critério com todas as alternativas de software em diferentes Matrizes de Avaliação. Para a elaboração das Matrizes foram descartados os critérios C10 e C11. Apesar das suas importâncias para a GI, esses critérios não foram reconhecidos em nenhuma das ferramentas selecionadas.

As pontuações atribuídas seguem o padrão proposto na Escala Fundamental. Assim, quando 
o software não atende o critério é atribuído o peso um, relativamente o mais baixo no MAH. Quando o software atende o critério é atribuído o peso nove, grau mais alto na Escala Fundamental.

Um vez criadas as Matrizes de Avaliação para cada um dos nove critérios estabelecidos, obteve-se o ranking dos softwares no apoio a GI. A tabela 3 apresenta este ranking com base na pontuação total obtida. Em seguida, as próximas colunas apresentam o nome do software, a pontuação total obtida e a quantidade de critérios atendidos. Pode-se observar que o software que obteve a maior pontuação foi o Innovation Suite.

Tabela 3: Ranking de softwares no apoio a GI

\begin{tabular}{c|c|c|c}
\hline Ranking & Software & Pontuação Total & Critérios Atendidos \\
\hline 1 & Innovation Suite & 1,90 & 7 \\
\hline 2 & Jenny & 1,40 & 6 \\
\hline 3 & Idea Ken & 1,15 & 5 \\
\hline 4 & Accept 360 & 1,10 & 3 \\
\hline 6 & Media Wiki & 0,85 & 3 \\
\hline 7 & Idea Box & 0,46 & 1 \\
\hline
\end{tabular}

\section{Análise dos Resultados e Conclusões}

Analisando o ranking (tabela 3) identifica-se que os softwares posicionados nas três primeiras posições possuem elevadas pontuações sobre os demais e atendem grande parte dos critérios estabelecidos. Innovation Suite, Jenny e Idea Ken são de fundamental relevância no apoio a GI. Esses softwares possuem características como a possibilidade de classificar a importância do conteúdo coletado, mecanismos de priorização de propostas e avaliação da viabilidade de ideias. Essas funcionalidades permitem aos softwares atuar como elemento central em etapas do processo inovativo como na Gestão de Ideias. Entre outras semelhanças se destaca ainda o fato de todos serem baseados em plataformas de serviços e utilizar tecnologias como a computação nas nuvens.

Entre os pontos de destaque observou-se que o Media Wiki foi o único software avaliado com potencial de explicitar, externalizar e socializar conhecimento organizacional. Merece citação também o Jenny, único produto com métodos para análise de gastos financeiros envolvidos no processo de inovação. Apenas Innovation Suite e Idea Ken apresentam recursos para o reconhecimento pela autoria de ideias e valorização intelectual ou financeira dos envolvidos. Esse quesito é destacado na manutenção e fomento do processo colaborativo [OECD 2005, Lodi, 2007].

Alguns softwares possuem a mesma quantidade de critérios atendidos mas ocupam posições diferentes no ranking (Jenny e Idea Ken, Media Wiki e Idea Box). A pontuação total superior obtida pelo Jenny e pelo Media Wiki se justifica pelo método de comparação aplicado. No MAH, quanto menor o conjunto de softwares que atendem a um critério, maior a pontuação atribuída a cada software. Apenas o Media Wiki atende o critério C1. Situação semelhante ocorre em análise dos critérios C8 e C9. Cada um desses critérios é atendido por apenas um software.

No contexto de avaliação identificou-se ainda que os critérios C5 e C6, referentes a capacidade de coleta e registro de ideias e ao compartilhamento e colaboração de ideias respectivamente, são os critérios atendidos por um maior número de softwares. Essa vocação da maioria dos softwares em criar um mecanismo de colaboração para aumentar a coleta de ideias com potencial inovador demonstra que grande parte dos softwares avaliados são de grande importância para a técnica de Inovação Aberta.

Por fim, o produto Innovation Suite merece atenção especial pelos resultados obtidos. Único 
capaz de controlar recursos e equipe de pessoas responsáveis pelo desenvolvimento das inovações, o software satisfaz com sucesso ainda aos critérios de monitoramento e integração entre empresa e ambiente interno/externo, de priorização de propostas de projetos de inovação, de avaliação da viabilidade de um projeto de inovação, de capacidade de coleta e registro de ideias, de compartilhamento e colaboração de ideias, de reconhecimento pela autoria de ideias e de suporte a alocação de recursos. Assim, nesse estudo, o software possui a melhor pontuação final entre os avaliados, ficando em primeiro lugar no ranking de softwares no apoio a GI.

Observou-se que a maior parte dos softwares é capaz de contribuir para a Inovação Aberta e para a Gestão de Ideias em conjunto. Innovation Suite, Jenny, Accept 360 e Idea Ken são exemplos de produtos que atendem a essas duas técnicas. A ferramenta Media Wiki, única a contemplar o critério relativo ao suporte a aquisição de conhecimento, permite explicitar e socializar o conhecimento organizacional e dessa forma contribui para o surgimento de inovações.

Responsáveis por auxiliar a técnica de Prospecção, InWeb e Sphinx se mostraram distantes dos processos de inovação. O InWeb é um projeto fechado que no momento não permite consultas a sua base de dados ou mesmo utilização de seus algoritmos. Já o Sphinx pode ser utilizado como instrumento auxiliar à técnica de Prospecção, atuando na coleta e classificação de dados.

Finalizando, observa-se que os critérios $\mathrm{C} 10$ e $\mathrm{C} 11$, referentes ao registro de acertos e erros em projetos e a capacidade de medir a difusão da inovação acabaram desconsiderados na aplicação do MAH. Isso se deve ao fato de que nenhum dos softwares avaliados atendeu a esses critérios. Para contemplar essas importantes lacunas do processo inovativo devem ser adotados softwares ou técnicas diferentes das analisadas nesse estudo.

\section{Referências}

Accept 360 Ideation (2012), http://www.accept360.com/solutions/accept360-ideation, Maio.

BrightIdea: The power of innovation. (2012) http://www.brightidea.com, Maio.

Cavalcanti, J. C. (2009) Gestão da Inovação com ênfase na Gestão de Tecnologias da Informação. In: 6TH International Conference on Information Systems and Technology Management. São Paulo: CONTECSI, 2009.

Cotec. (1998) Temaguide: a Guide to Technology Management and Innovation for companies. Barcelona: Cotec.

Ideabox. (2012) The Web Based Suggestion Box. http://ideabox.phpoutsourcing.com/index.php, Maio.

Ideaken. (2012) http://www.ideaken.com, Agosto.

InWeb (2012) Instituto Nacional de Ciência e Tecnologia para a Web, http://www.inweb.org.br/inweb, Junho.

JPB. (2010) Jenny Innovation Proccess Management: IPM, http://www.jpb.com/jenni/index.php, Outubro.

Limas, C. E. A.; Scandelari, L.; Franciso, A. C. (2008) O Uso das Ferramentas da Tecnologia nas Pequenas e Microempresas de Ponta Grossa - PR. In: Encontro de Engenharia e Tecnologia dos Campos Gerais, 2, Ponta Grossa, Paraná.

Lodi, I.E. (2006) NUGIN: Núcleo de Apoio ao Planejamento e Gestão da Inovação, http://www.propesquisa.ufsc.br/arquivos/IEL-SC-NUGIN-Eliza-2006.pdf, Outubro.

MediaWiki. (2011) http://www.mediawiki.org/wiki/MediaWiki, Junho.

Moraes, E.A.; Santaliestra, R. (2008) Modelo de decisão com múltiplos critérios para escolha de software de código aberto e software de código fechado. Organizações em contexto, Ano 4, n. 7.

Nonaka, I.; Takeuchi, H. (2008) Gestão do Conhecimento. 1. ed. Porto Alegre: Bookman.

OECD (2005) Organization for Economic Co-operation and Development. Oslo Manual: Proposed guidelines for collecting and interpreting innovation data. 3.ed. European Comission: OECD, www.oecd.org/dataoecd/35/61/2367580.pdf, Outubro.

Sphinx (2010) http://www.sphinxbrasil.com, Novembro. 